\title{
The need for better pre-hospital analgesia
}

\author{
J. A. CHAMBERS \& H. R. GULY
}

Accident and Emergency Department, Derriford Hospital, Plymouth

\section{SUMMARY}

Many patients arrive at the accident and emergency (A\&E) department in pain. To quantify this problem a retrospective analysis was performed of the clinical records of 502 consecutive patients arriving by ambulance at the A\&E department over a 20-day period. A total of $273(54 \%)$ of the patients had pain as a symptom on arrival and $69(14 \%)$ were given opioid analgesia in the A\&E department. Sixty of those given opioids had a single limb fracture. A survey of all ambulance services in the U.K. was conducted by means of a postal questionnaire sent to chief ambulance officers. Only five out of 65 services give any analgesia other than Entonox (B.O.C.). There were wide variations in the attitudes of services around the country to future developments. The authors suggest that paramedics should be trained to administer intravenous opioid analgesia.

\section{INTRODUCTION}

Many patients with injuries and some with non-traumatic problems including myocardial infarction are brought by ambulance to the A\&E department in pain. To discover the frequency of pain as a symptom, retrospective review of patients clinical records was performed. In the United States pre-hospital intravenous analgesia has been given by paramedics under physician control (Stene et al., 1988). Although there is no published data, the authors are aware of some work in this country using intravenous nalbuphine hydrochloride administered by ambulance personnel. As a subsidiary study a postal questionnaire of all chief ambulance officers was undertaken to discover what methods of analgesia are currently in use in the U.K.

Correspondence: $\mathrm{Mr}$ J. A. Chambers, Director of Emergency Medicine, Dunedin Hospital, Dunedin, Nezo Zealand. 


\section{METHODS}

\section{Pain survey}

The authors hoped to conduct a prospective study to assess pain suffered by patients during the transport phase to hospital and in the A\&E department using visual analogue scores. A pilot study demonstrated significant problems in data collection. Both staff and patients had trouble using the scaling system and less than $30 \%$ of ambulance arrivals had complete information. Therefore, to ensure that figures reflected the complete picture, a retrospective analysis of the clinical notes of all patients arriving by ambulance at an A\&E department over a 20-day period was performed. Information regarding the patients' age and diagnosis was recorded along with written evidence of the presence of 'pain' on arrival and any analgesic therapy. In those who received opioids, notes were made of clinical factors which should lead to caution but not preclude the use of such analgesia (Table 1) and any adverse effects were documented.

\section{Survey of present analgesic practice}

A questionnaire was sent to all 65 chief ambulance officers in the U.K. Details of present practice and future developments were sought.

\section{RESULTS}

\section{Pain survey}

Of 502 patients arriving by ambulance $340(68 \%)$ had sustained trauma and 162 (32\%) had a non-traumatic complaint. Figure 1 shows the numbers of patients in pain. $69(14 \%)$ patients were given opioid analgesia in the A\&E department. Five

Table 1. Risk factors sought in those patients given opioid analgesia in A\&E

Clinical factors increasing the risk of adverse effects when using opioid drugs

$\begin{aligned} & \text { Extremes of age } \quad<10 \text { years } \\ & >75 \text { years }\end{aligned}$
Intoxication
Head injury with history of unconsciousness
Chest injury
Abdominal injury
Documented hypotension
Documented hypoxia




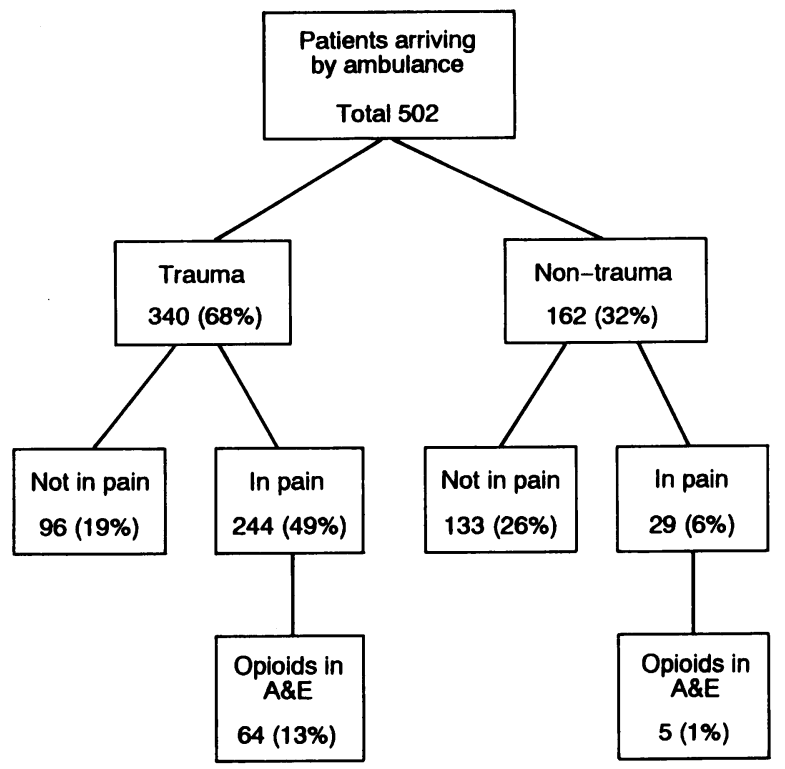

Fig. 1. Number of patients arriving by ambulance in pain at A\&E over a 20-day period.

of these patients had severe chest pain. The remainder had sustained burns, fractures or dislocations - in 60 cases a limb fracture. Thirty-five who received opioids had risk factors. One was a child aged 7 with multiple fractures, five had associated head injuries and two had associated chest injuries. Twenty-seven patients were aged over 75 years of whom 20 had hip fractures. None of these patients developed serious side effects after opioid administration. Diamorphine, pethidine and nalbuphine were the agents administered by the A\&E medical staff.

\section{Ambulance service survey}

Sixty-three out of 65 questionnaires (97\%) were returned, usually after delegation to a senior training officer. Entonox is used by all ambulance services in the U.K. Three ambulance services at present use nalbuphine and in one of these it is limited to a trial of administration in patients with chest pain. All three use doses of $10-20 \mathrm{mg}$ given intravenously and follow simple protocols. Only trained paramedics are allowed to administer the drug and no medical consultation is required. All three services made positive comments with no reports of serious sequelae. A follow-up communication from one service suggested that they were considering giving larger doses to some patients to achieve better analgesia. One service is using diclofenac by intramuscular (I.M.) injection. Another rural service is conducting a trial using diamorphine in patients with chest pain in cooperation with a local consultant cardiologist.

One third of respondents, whilst using only Entonox at present, expressed an 
interest in other analgesics. Most mentioned that they were awaiting expected legislation regarding the use of Prescription Only Medicines before making changes $\_$ to present regimes. Two services replied that after consideration of the practicalities and dangers by their medical advisory groups they could see 'no foreseeable date' when injected analgesics would be introduced. A number of services noted that GPs offered good back up in circumstances where analgesia other than Entonox was required.

\section{DISCUSSION}

The relief of pain is one of the priorities of emergency treatment. The humanitarian needs are obvious and for many years morphine has been issued to soldiers for self-administration in times of war and to non-medical emergency services such ${ }_{\dot{\omega}}$ mountain rescue teams and the RNLI.

The benefits of analgesia however extend beyond relief of symptoms. In acute myocardial infarction opioid analgesia has been shown experimentally to reduce the incidence of life threatening arrythmias (Fagbemi et al., 1983). In clinical $\vec{\infty}$ practice, pre-hospital coronary care, of which adequate opioid analgesia was a $\frac{\mathbb{O}}{0}$ major component, has been shown to reduce community mortality (Mathewson $\stackrel{\mathbb{D}}{3}$ et al., 1985). In trauma victims good treatment and resuscitation during the 'Golde Hour' is regarded as vital to improving survival (Commission on the Provision $\overrightarrow{0}$ Surgical Service, 1988). Good analgesia reduces shock and in general makes patie management easier, improving tolerance of and compliance with therapeuts manoeuvres such as extrication from wreckage, administration of oxygen and application of splints.

The administration of opioid analgesia in the A\&E department implies that

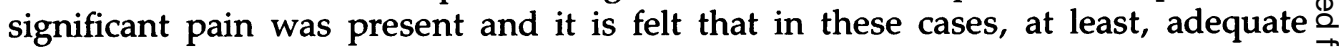
pain relief could usefully have been provided earlier i.e., in the pre-hospital phase $\overrightarrow{\vec{\sigma}}$ of care. In this study few A\&E ambulance arrivals had chest pain. This reflects the local primary care system. Patients with suspected myocardial infarction can, after treatment at home by the GP, be admitted directly to the coronary care unit. Of those who were given opioid analgesia the majority had sustained trauma and $\stackrel{3}{\underline{-}}$. most of these had a single limb fracture. Whilst a reason for caution was present in $\overline{3}$ half of those receiving opioids there were no clinical problems in the A\&E department. The major reason for caution was extreme age in patients who had most commonly suffered a fracture of the proximal femur.

Entonox (B.O.C.) has been the analgesic used by the ambulance services since $>$

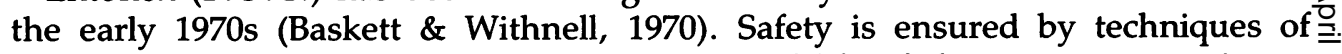
patient driven administration and the short half life of the active agent nitrous $N$ oxide. Analgesia is not always optimal and patient compliance is, at times, poor. $N$ Patients with painful limb fractures nowadays express disbelief when the paramedic N who skillfully inserts an intravenous cannula injects only saline and offers 'gas tog breathe'. The intramuscular route of administration of analgesia is unreliable ando hazardous in the shocked patient and for these reasons, if no other, I.M. diclofenac is not an ideal analgesic. 
Head, chest and abdominal injury have traditionally been stated as contraindications to opioid analgesia. In head injury opioids may affect conscious level, the pupillary response and, by causing respiratory depression and hypercarbia, raise intracranial pressure (Jaffe \& Martin, 1985). However, pain also increases intracranial pressure and head injury is not an absolute contraindication to adequate analgesia. Respiratory depression could worsen hypoxia caused by a chest injury but in practice reducing pain on chest wall movement can improve the depth of respiration and hence oxygenation. Abdominal pain has been felt to mask signs of peritoneal irritation. In practice opioid analgesia titrated carefully against pain relaxes the patient and makes the demonstration of true signs more straightforward (and most patients with suspected abdominal injury after trauma will be investigated using ultrasound, computerized tomography or diagnostic peritoneal lavage). The presence of the above injuries does introduce the potential for adverse effects following opioid administration. It is felt that, in the pre-hospital phase injury to the chest, head or abdomen should be reasons for exclusion or deferral until a doctor arrives on scene. This study shows that even if protocols restricted opioid analgesia administration by paramedics to those with limb fractures, $87 \%$ of those in significant pain might benefit.

Legal problems have been overcome to allow rescue teams to carry morphine but more widespread introduction does raise problems of security. A synthetic morphinian agent such as nalbuphine hydrochloride may offer a solution. It is not a controlled drug but is a Prescription Only Medicine and it causes less respiratory depression than morphine and has little psychomimetic effect (Gal et al., 1982). It is of little benefit to the opioid drug abuser expecting relief from withdrawal symptoms (Graham \& Lewis, 1977). In a pre-hospital setting its successful and safe use has been documented by paramedics treating trauma patients (Stene et al., 1988) and junior cardiologists treating cardiac pain (Troughton \& Adgey, 1989).

The ambulance service of today possess many more skills and are increasingly better qualified than those of former years. No longer do they simply 'scoop and run'. Many developments have, rightly, been made in the treatment of life-threatening emergencies. Ambulance personnel are skilled at resuscitation and administer potent drugs such as atropine, adrenaline and salbutamol. The provision of strong and effective analgesia would extend the role of the service in terms of quality of care - relieving a symptom not necessarily life-threatening but always distressing. The $97 \%$ response rate to our questionnaire demonstrates the interest of those who deal, on a daily basis, with those patients in pain and many requested feedback from our findings. At present few ambulance services are using strong analgesics, and some consider such an option fraught with danger.

Inconsistency in the attitudes of individual ambulance services is not surprising as they remain largely independent of each other and reliant on local medical advice on matters related to extended training (Weston et al., 1990). Patients should not have to wait until they arrive in the A\&E department to obtain adequate pain relief. We suggest that paramedics should be allowed to administer opioid analgesia using protocols for patients with limb fractures, burns and ischaemic cardiac pain. 


\section{REFERENCES}

Baskett P. J. F. \& Withnell A. (1970) Use of entonox in the ambulance service. British Medical Journa?

2, 41-43.
Commission on the Provision of Surgical Services. (1988) The Management of Patients with Majo $\frac{\overrightarrow{\bar{N}}}{F}$ Injuries. The Royal College of Surgeons of England.

Fagbemi O., Kane K. A., Lepran I., Parratt J. R. \& Szerkeres L. (1983) Antiarrythmic actions o meptazinol, a partial agonist at opiate receptors, in acute myocardial ischaemia. British Journal o Pharmacology 78, 455-460.

Gal T. J., DiFazio C. A. \& Moscicki J. (1982) Analgesic and respiratory depressant activity of nalbuphineڤึ A comparison with morphine. Anaesthesiology 57, 367-374.

Graham J. D. P. \& Lewis J. W. (1977) The assessment of abuse potential of drugs of the opiate type. In:Pain (eds Harcus A. W., Smith R. \& Whittle B.), pp. 84-96. Churchill Livingstone, Edinburgh.

Jaffe J. H. \& Martin W. R. (1985) Opioid analgesics and antagonists. In: Goodman and Gilman's Tho Pharmacological Basis of Therapeutics, ed 7 (eds Gilman A. G., Goodman L. S., Rall T. W. et al.) B. pp. 502. Macmillan, New York.

Mathewson Z. M., Mcloskey B. G., Evans A. E., Russell C. J. \& Wilson C. (1985) Mobile coronary care and community mortality from myocardial infarction. Lancet $i, 441$.

Stene J. K., Stofberg L., Macdonald G., Myers R. A., Ramzy A. \& Burns B. (1988) Nalbuphine analgesiå in a pre-hospital setting. American Journal of Emergency Medicine 6, 634-639.

Troughton T. G. \& Adgey A. A. J. (1989) High dose nalbuphine in acute myocardial infarction International Journal of Cardiology 23, 53-57.

Weston C., Goodall K. \& Stevens M. (1990) Variations in the provision of extended trained ambulance् personnel within the Welsh ambulance service. Health Trends 3, 121-4. 